\title{
Study on Diesel Engine Fault Diagnosis Method based on Integration Super Parent One Dependence Estimator
}

\author{
Wang Xin \\ College of Marine Engineering, Dalian Maritime University, Dalian, China \\ bfddwx@163.com \\ Yu Hongliang \\ College of Marine Engineering, Dalian Maritime University, Dalian, China \\ Yhl1202@dl.cn \\ Zhang Lin \\ School of Textile and Light Industry, Dalian Polytechnic University, Dalian, China \\ zhlpopo@126.com \\ Huang Chaoming \\ College of Marine Engineering, Dalian Maritime University, Dalian, China \\ hchaoming@163.com \\ Song Yuchao \\ College of Marine Engineering, Dalian Maritime University, Dalian, China \\ 47443532@qq.com
}

\begin{abstract}
Under the background of the deficiencies and shortcomings in traditional diesel engine fault diagnostic, the naïve Bayesian classifier method which built on the basis of the probability density function is adopted to diagnose the fault of diesel engine. A new approach is proposed to weight the super-parent one dependence estimators. To verify the validity of the proposed method, the experiments are performed using 16 datasets collected by University of California Irvine (UCI) and 5 diesel engine datasets collected by our lab. The comparison experimental results with other algorithms demonstrate the effectiveness of the proposed method.
\end{abstract}

Index Terms-diesel engine; naïve Bayesian classifier; fault diagnosis; one-dependence classifier

\section{INTRODUCTION}

Diesel engine is a complex machine and a multi-interference system. The relationship between its input and output variables, fault and sign is unobvious and uncertainty. Poor working conditions easily lead to signal distortion etc. These have greatly increased the types of diesel engine fault diagnosis difficulty. In recent years, scholars from various countries for the diesel engine fault diagnosis methods have made a lot of related.

Bayesian diagnosis is established based on the probability density function. Compared to the diagnosis based on the failure mechanism, it has smaller diagnostic error rates. So it has an extensive application. With the development of information and automation technology, a lot of running data and diagnostic data has been accumulated and it is possible to calculate the prior probabilities of Bayesian method. However, in many practical fields, the independency assumption of Naïve Bayes (NB) does not hold. Therefore, many researches are mainly about how to use some technology to find a most favorable topology among all possible network structures. At present, such technologies can be summarized into two categories: heuristic search and correlation analysis. To relax the independency assumption, the researchers have done a lot of work. With the development of modern science technology and the degree of automation, diesel engine fault diagnostic technique has undergone significant changes and Bayesian diagnosis become one of the most efficient diagnosis methods.

Among all these improving approaches, One-Dependence Estimators (ODEs) ${ }^{[1]}$ are simple but effective classifiers. ODEs are very similar to NB but they also allow every attribute to depend on, at most, another attribute besides the class. Both theoretical analysis and empirical evidence have shown that ODEs can improve upon NB's accuracy when its attribute independence assumption is violated. Tree Augmented Bayesian Network (TAN) ${ }^{[2]}$ is kind of ODE which provides a powerful alternative to NB. Super Parent-One-Dependence Estimators (SPODEs) ${ }^{[3]}$ can be considered a subcategory of ODEs where all attributes depend on the same attribute. L.X. Jiang ${ }^{[4]}$ et al adds the parent node for some attributes in Bayesian network using conditional mutual information. Aggregating One-Dependence Estimators (AODE) ensembles all SPODEs that satisfy a minimum support constraint [5] and estimate class conditional probabilities by averaging across them. This ensemble has demonstrated very high prediction accuracy with modest computational requirements. However, it is based on an implicit assumption that all SPODEs have the same or equivalent learning ability. But the leaning ability of different Bayesian networks is different. Simply averaging all SPODEs may scale up the influence of the bad performance classifiers so as to 
affect the final classification result. WAODE [6] is an improvement of AODE. It uses conditional mutual information to determine the weight of each SPODE. HNB [7] and HODE [8] are another two improved versions of AODE. Addressing how to select SPODEs for ensemble so as to minimize classification error, Yang $\mathrm{Y}$ et al [9] proposed five selection methods, minimum description length (MDL), minimum message length (MML), and leave one out (LOO), Backward Sequential Elimination (BSE) and Forward Sequential Addition (FSA). Their experimental results showed that measuring ensembles outperforms measuring single SPODE and model selection for SPODE is advisable since the selection makes differences. In addition, Li Nan et al [10] take each SPODE as a production model and weight each SPODE using the fitting degree of the model to data.

\section{SUPERPARENT-ONE-DEPENDENCE ESTIMATORS (SPODES)}

Assume $\mathrm{D}$ is a set of training instances, $A=\left\{A_{1}, \ldots A_{n}\right\}$ is the attributes variable set, where $\mathrm{N}$ is the number of attributes, $C$ is a class variable, $C$ is a value of $\mathrm{C}$. $a_{1}, \cdots, a_{i}, \cdots, a_{n}$ are the attribute value of $A_{1}, \cdots, A_{i}, \cdots, A_{n}$ respectively.

A SPODE requires all attributes to depend on the same attribute, namely the super parent, in addition to the class. Let $S P O D E_{A_{t}}$ denote the SPODE with super parent At. $S P O D E_{A_{t}}$ will estimate the probability of each class label $C$ given an instance $X$ as follows:

$$
P(c \mid X)=\frac{P(c, X)}{P(X)}=\frac{P\left(c, a_{t}\right) \prod_{j=1, j \neq t}^{n} P\left(a_{j} \mid c, a_{t}\right)}{P(X)}
$$

Since the above equality holds for every SPODE, it also holds for the mean over any subset. An ensemble of $\mathrm{k}$ SPODEs corresponding to the super-parents $A_{1}, \cdots, A_{k}$ estimates the class probability by averaging their results as follows.

$$
P(c \mid X)=\frac{\sum_{i=1}^{k} P\left(c, a_{i}\right) \prod_{j \neq i} P\left(a_{j} \mid c, a_{i}\right)}{k \times P(X)}
$$

AODE selects a limited class of 1-dependence classifiers and aggregate the predictions of all qualified classifiers within this class. To avoid including models for which the base probability estimates are inaccurate, ensemble of all SPODEs except for those who have less than 30 training instances. Hence, AODE classifies an instance $X$ by using the following equality.

$$
P(c \mid X)=\frac{\sum_{i: 1 \leq i \leq n \wedge F\left(a_{i}\right) \geq m} \hat{P}\left(c, a_{i}\right) \prod_{j=1}^{n} \hat{P}\left(a_{j} \mid c, a_{i}\right)}{\left|1 \leq i \leq n \wedge F\left(a_{i}\right) \geq m\right| \times P(X)}
$$

Where $\mathrm{F}\left({ }^{a_{i}}\right)$ is a count of the number of training examples having attribute-value $a_{i}$ and is used to enforce the limit $\mathrm{m}$ that we place on the support needed in order to accept a conditional probability estimate. In the presence of estimation error, if the inaccuracies of the estimates are unbiased the mean can be expected to factor out that error.

\section{NEW APPROACH TO WEIGHT SPODE}

A data sample of $n$ attributes can potentially have $\mathrm{n}$ SPODEs, each alternatively taking a different attributes as the super parent, as shown in Fig.1. In this paper, we consider the diversity of SPODE and its corresponding NB and propose a new approach to weight SPODE.

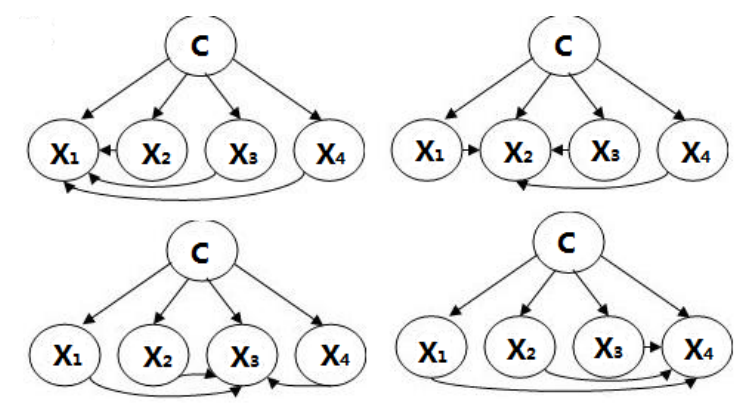

Fig.1. 4 SPODEs with 4 attributes

A. Diversity of SPODE and its corresponding NB

A natural question is that how well a SPODE can perform in predictive tests. If the prediction result for a test instance by using a SPODE is same with that by using NB, we can say that the performance of the SPODE is not very well. We would rather use its corresponding NB than the SPODE since the structure of SPODE is more complex than simple NB and the parameter estimation needs more time while the performance are the same.

Definition 1 If a test instance $X$ is classified to the same class by using SPODE and by using NB, then we say the performance of SPODE and NB is equivalence.

Definition 2 Suppose there are $\mathrm{m}$ classes. Let Diver $\left(S P O D E_{A_{t}}, N B\right)$ be a $m \times m$ square array such that Diver $_{i j}$ is the number of test examples assigned to class $c_{i}$ by $S P O D E_{A_{t}}$ with and to class ${ }^{c_{j}}$ by its 
corresponding NB. Let $\mid$ Diver $\mid$ be the number of all test examples. The diversity of ${ }^{S P O D E_{A_{t}}}$ and its corresponding NB is defined as follows.

$$
\operatorname{Diff}\left(A_{t}\right)=\frac{1-\alpha}{1-\beta}
$$

Where $\alpha$ be the probability that the $S P O D E_{A_{t}}$ and its corresponding NB perform the same, $\beta$ be the probability that the $S P O D E_{A_{t}}$ and its corresponding NB agree by chance. They are respectively defined as follows.

$$
\begin{aligned}
& \alpha=\frac{\sum_{i=j=1}^{m} \text { Diver }_{i j}}{\mid \text { Diver }} \\
& \beta=\sum_{i=1}^{m}\left(\sum_{j=1}^{m} \frac{\text { Diver }_{i j}}{\mid \text { Diver }_{j=1}^{m}} \sum_{j=\text { Diver }_{j i}}^{\mid \text {Diver }}\right)
\end{aligned}
$$

Further, we have the following discussion.

When $\operatorname{Diff}\left(A_{t}\right)=0$, we can get $\alpha=1$. It means that the classification results using $S P O D E_{A_{t}}$ and its corresponding NB are completely same. The two classifiers agree on every example.

When $\operatorname{Diff}\left(A_{t}\right)=1$, we can get $a=\beta$. It means that $S P O D E_{\mathrm{A}_{t}}$ and its corresponding NB equals that expected by chance.

When $\operatorname{Diff}\left(A_{t}\right)>1$, we can get $a<\beta$. It means that agreement is weaker than expected by chance. In another words, the chance of the two classifiers obtain the same classification is slim.

If the diversity between the augmented naïve bayes and the simple naïve bayes is small, it has no need to expand the network structure of NB. Because of the complexity of probability estimation is closely related to the network structure.

From the above discussion, we can get the conclusion that the diversity between the augmented naïve bays $S P O D E_{A_{t}}$ and the simple naïve bays increases with the increase of the value of $\operatorname{Diff}(\mathrm{At})$. Therefore, we take $\operatorname{Diff}\left(A_{t}\right)$ as the weight of $S P O D E_{A_{t}}$.

\section{B. SPODEs Ensemble}

According to the above discussion, firstly we give the definition of the weight of $S P O D E_{A_{t}}$.

Definition 3 Let $\omega_{t}$ represents the weight of $S P O D E_{A_{t}} . w_{t}$ is defines as:

$$
\omega_{t}=\frac{\operatorname{Diff}\left(A_{t}\right)}{\sum_{i=1}^{n} \operatorname{Diff}\left(A_{i}\right)}\left(\sum_{i=1}^{n} \operatorname{Diff}\left(A_{i}\right) \neq 0\right)
$$

Where $\operatorname{Diff}\left(A_{t}\right)$ is defined in (4) and $\sum_{t=1}^{n} \omega_{t}=1$.

Since (1) holds for every SPODE, we can estimate the probability of each class label ${ }^{C}$ given an instance $X$ as follows:

$$
P(c \mid X)=\left\{\begin{array}{c}
\frac{\sum_{t=1}^{n} \omega_{t} P\left(c, a_{t}\right) \prod_{j=1, j \neq t}^{n} P\left(a_{j} \mid c, a_{t}\right)}{P(X)}, \sum_{t=1}^{n} \omega_{t} \neq 0 \\
\frac{P(c) \prod_{j=1}^{n} P\left(a_{j} \mid c\right)}{P(X)}, \text { else }
\end{array}\right.
$$

The proposed classifier is named WSPODE. WSPODE classifies a newly instance using (9) as well.

$$
\arg \max _{c} P(c \mid X)
$$

The value of $w_{t}$ can determine the number of SPODEs used as well as the importance of every SPODE. For example, if $w_{t}=0$ and $w_{j} \neq 0(j \in[1, n], j \neq t)$, we actually only select $n-1$ SPODEs. In a extreme case $\sum_{\text {when }} \sum_{i=1}^{n} \operatorname{Diff}\left(A_{i}\right)=0$, we will use NB to classify all test samples. But this kind of situation is rare. Because it means that all SPODE perform exactly the same with NB. Every SPODE and its corresponding NB agree on every example.

\section{ALGORITHM DESCRIPTION}

In this section, we describe our algorithm for training and inference. During the training phase, the goal is to determine the weight of every SPODE using (7). The learning algorithm for WSPODE is depicted as follows. In classification phase, use (8) to classify a newly instance without class label. 
Algorithm. WSPODE (D)

Training phase:

Input: a set $\mathrm{D}$ of training examples

Output: a Improved Naive Bayesian

Classifier for D

Using NB to classify training examples in $\mathrm{D}$ and store the result;

For each attribute At $(t=1, \ldots, n)$

There is a Diver[m][m], where $\mathrm{m}$ is the number of class labels; class label

For each example 1 in $\mathrm{D}$ delete its

$$
\begin{gathered}
\operatorname{SPODE}_{\mathrm{A}_{\mathrm{t}}} \text { and to } c_{k} \text { by NB }(\mathrm{j}, \mathrm{k}=0, \ldots, \mathrm{m}-1) \\
\text { Then Diver[j][k]= Diver[j][k]+ } \\
\text { For } \mathrm{j}=0 \text { to } \mathrm{m}-1, \mathrm{k}=0 \text { to } \mathrm{m}-1 \\
\text { Diver[j][k] = Diver[j][k]/the size of D; } \\
\text { Compute } \alpha \text { using (5); } \\
\text { Compute } \beta \text { using (6); } \\
\text { Compute Diff(At) using (4); } \\
\text { Compute } \omega_{t} \text { using (7); }
\end{gathered}
$$$$
\text { If } 1 \text { is classified to } c_{j} \text { by }
$$$$
\text { Then Diver }[\mathrm{j}][\mathrm{k}]=\operatorname{Diver}[\mathrm{j}][\mathrm{k}]+1 \text {; }
$$$$
\text { For } \mathrm{j}=0 \text { to } \mathrm{m}-1, \quad \mathrm{k}=0 \text { to } \mathrm{m}-1
$$

Sum $=0$;

For $\mathrm{i}=1$ to $\mathrm{n}$ sum $=\operatorname{sum}^{+}{ }^{\omega_{i}}$;

For $\mathrm{i}=1$ to $\mathrm{n} \omega_{i}=\omega_{i} /$ sum;

Classification phase:

Input: the Improved Classifier built in training phase, a newly instance $\mathrm{X}$

Output: the class label $\mathrm{c}$ of $\mathrm{X}$

For $\mathrm{i}=1$ to $\mathrm{m}$

Compute $\mathrm{P}(\mathrm{ci} \mid \mathrm{X})$ using (8), the parameters are obtained in training phase;

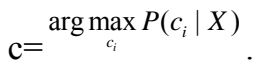

Assume that the number of training instances and attributes are $\mathrm{s}$ and $\mathrm{n}$ respectively. The number of classes is $\mathrm{m}$. The average number of values for an attributes is $\mathrm{v}$.

In order to train the weight of every SPODE, we compute Diver $\left(S P O D E_{A_{t}}, N B\right)$ for each $S P O D E_{A_{t}}$ using the training data without the class label. Hence, compared to AODE, it needs more $O(s m n)$ time complexity.

In classification phase, the time complexity of the WSPODE is o(mn2). It is just the same as AODE.

TableI. Time Complexity

\begin{tabular}{ccc}
\hline algorithm & Training & classification \\
\hline AODE & $o\left(m n^{2}\right)$ & $o\left(m n^{2}\right)$ \\
WAODE & $o\left(s n^{2}+n\right)$ & $o\left(m n^{2}\right)$ \\
LODE & $o\left(s n^{2}+s n\right)$ & $o\left(m n^{2}\right)$ \\
WSPODE & $o\left(s n^{2}+s m n\right)$ & $o\left(m n^{2}\right)$ \\
\hline
\end{tabular}

\section{EXPERIMENTS}

\section{A. Experimental settings}

(1) Test data

In this paper, we choose $16 \mathrm{UCI}$ datasets (shown in table II ) and 3 foul diagnostic datasets of diesel engine (shown in tableIII). WD615 diesel engine valve fault diagnosis data is used to de simulation experimental.

The datasets of diesel engine is got by The Dewetron Combustion Analyzer as fig2-5. The Dewetron Combustion Analyzer systems are used for engine research, development and optimization. Also for component development and testing, such as ignition systems, exhaust systems and valve control gear. Through measuring diesel engine cylinder pressure and crank angle, eigenvalues are calculated to analysis.

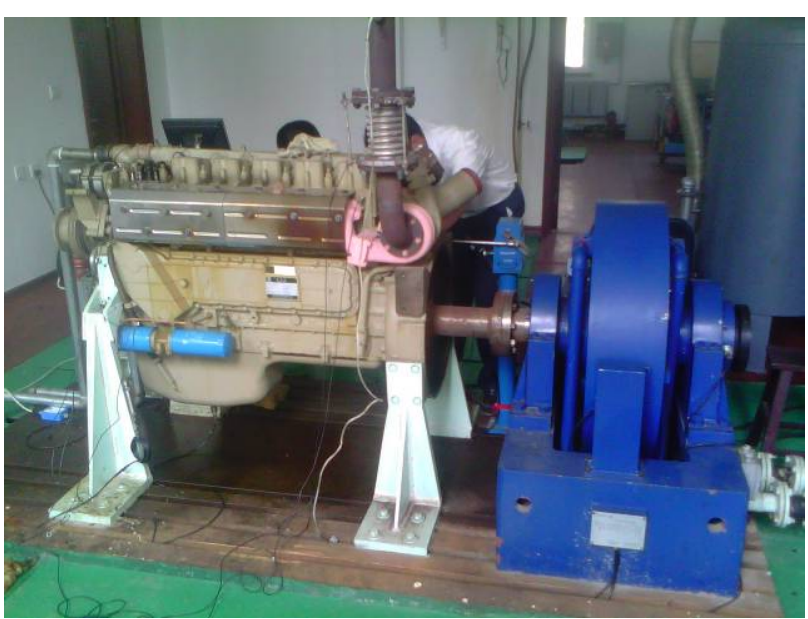

Figure.2. WD615 diesel engine

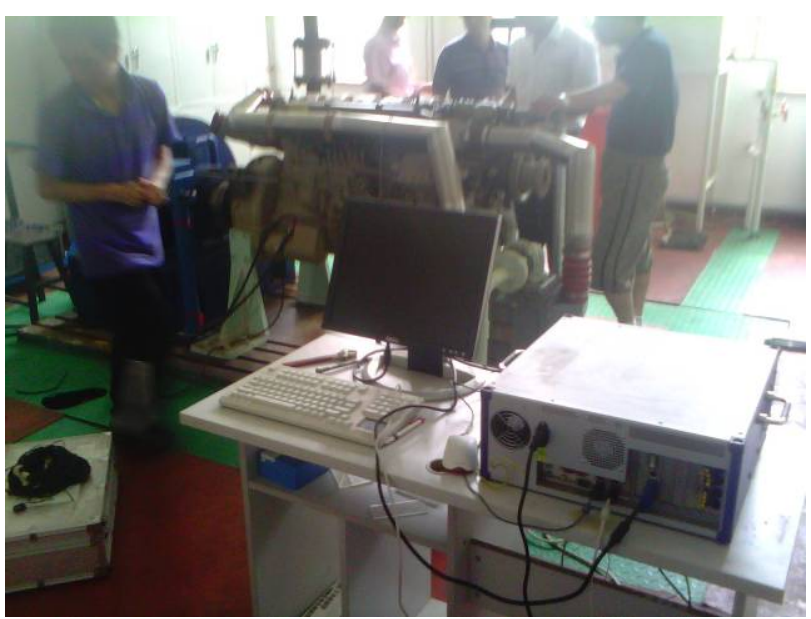

Fig.3. Dewetron Combustion Analyzer 


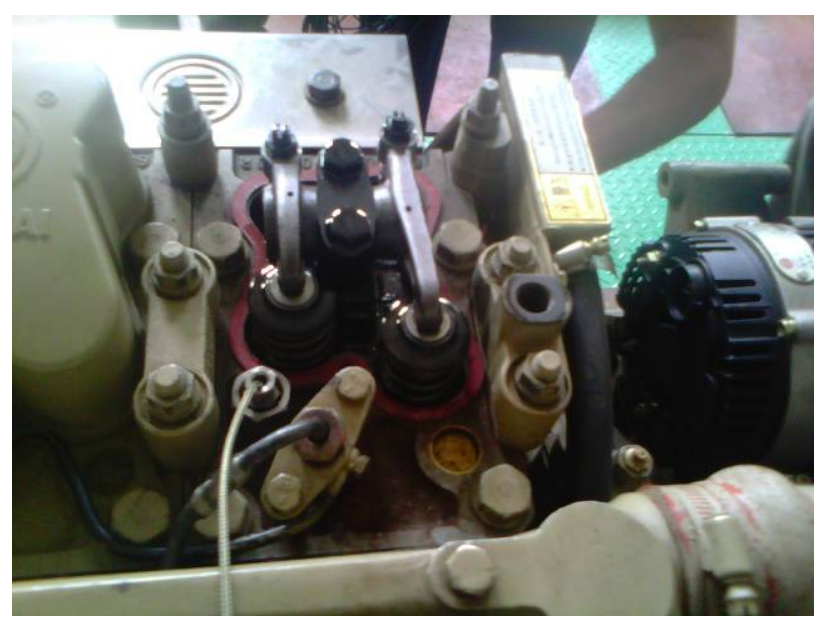

Fig.4. Pressure Sensor

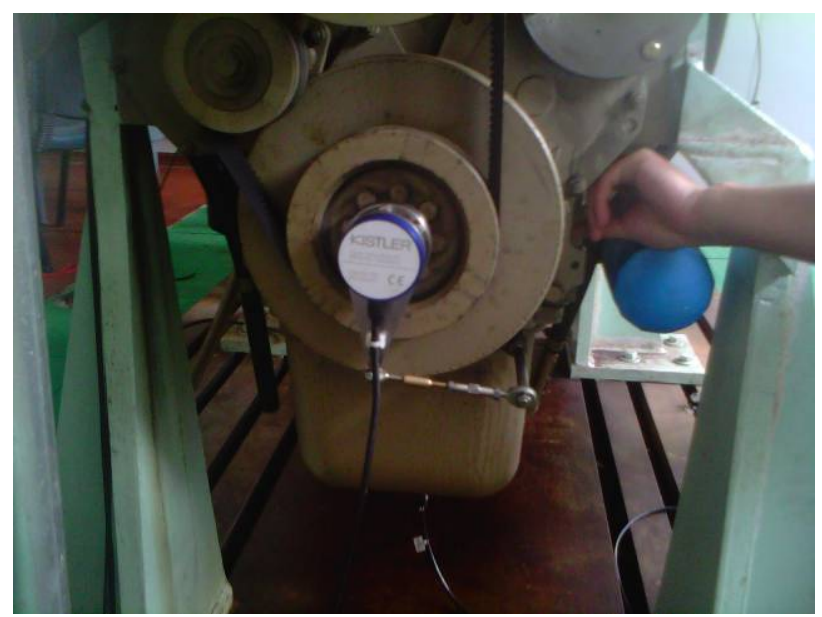

Fig.5. Crank Angle Sensor

All datasets are preprocessed with the help of WEKA1 software before using. Use the supervised filter Discretize in WEKA to discretize all the numeric attributes; Use the unsupervised filter Remove in WEKA to remove useless attributes. The discretized data is showed in tableIV.

\begin{tabular}{|c|c|c|c|c|c|c|c|c|c|}
\hline & & $\mathrm{Tab}$ & & & da & sets & & & \\
\hline $\begin{array}{l}\text { I } \\
\text { d }\end{array}$ & dataset & size & $\begin{array}{l}\mathrm{C} \\
\#\end{array}$ & $\begin{array}{l}\text { A } \\
\#\end{array}$ & Id & dataset & size & $\begin{array}{l}\mathrm{C} \\
\#\end{array}$ & $\begin{array}{l}\text { A } \\
\#\end{array}$ \\
\hline 1 & $\begin{array}{c}\text { breast_ } \\
\text { w }\end{array}$ & 699 & 2 & 9 & 9 & machine & 209 & 7 & 7 \\
\hline 2 & car & $\begin{array}{c}172 \\
8\end{array}$ & 4 & 6 & $\begin{array}{l}1 \\
0\end{array}$ & promoter & 106 & 2 & 57 \\
\hline 3 & $\begin{array}{c}\text { diabete } \\
\mathrm{s}\end{array}$ & 768 & 2 & 6 & $\begin{array}{l}1 \\
1\end{array}$ & sonar & 208 & 2 & 21 \\
\hline 4 & Flags & 194 & 6 & 30 & $\begin{array}{l}1 \\
2\end{array}$ & $\underset{\text { oe }}{\text { tic_tac_t }}$ & 958 & 2 & 9 \\
\hline 5 & flare & $\begin{array}{c}138 \\
9\end{array}$ & 2 & 13 & $\begin{array}{l}1 \\
3\end{array}$ & vote & 435 & 2 & 16 \\
\hline 6 & heart_h & 294 & 5 & 9 & $\begin{array}{l}1 \\
4\end{array}$ & vowel & 990 & $\begin{array}{l}1 \\
1\end{array}$ & 14 \\
\hline 7 & iris & 150 & 3 & 4 & $\begin{array}{l}1 \\
5\end{array}$ & $\begin{array}{c}\text { wavefor } \\
\mathrm{m}\end{array}$ & $\begin{array}{c}500 \\
0\end{array}$ & 3 & 19 \\
\hline 8 & lymph & 148 & 4 & 18 & $\begin{array}{l}1 \\
6 \\
\end{array}$ & zoo & 101 & 7 & 16 \\
\hline
\end{tabular}

$\overline{1} \mathrm{http}: / /$ www.cs.waikato.ac.nz/ml/weka/

Copyright (C) 2011 MECS
TableIII. Diesel datasets

\begin{tabular}{|c|c|c|}
\hline & number & Description \\
\hline size & 1600 & $\begin{array}{l}\text { When the load is } 0 \mathrm{Nm}, 100 \mathrm{Nm}, 200 \mathrm{Nm}, 300 \mathrm{Nm} \\
\text { and } 400 \mathrm{Nm} \text {, collect } 2000 \text { cycles' data } \\
\text { respectively. The datasets are denoted as Diesel1, } \\
\text { Diesel2, Diesel3, Diesel4 and Diesel5. Each } \\
\text { dataset has } 2000 \text { records. }\end{array}$ \\
\hline .A\# & 12 & $\begin{array}{l}\text { (1) speed; (2) maximum Cylinder Pressure; (3)phase } \\
\text { of maximum Cylinder Pressure; (4)phase of } \\
\text { maximum of rising rate of maximum Cylinder } \\
\text { Pressure; (5)phase when energy conversion reaches } \\
50 \% \text {; 6) phase of starting burning,; (7)phase of } \\
\text { ending burning; (8)phase difference between } \\
\text { burning start and burning end; (9) mean indicating } \\
\text { effective pressure; (10) net mean effective pressure; } \\
\text { (11)indicated power }\end{array}$ \\
\hline $\mathrm{C} \#$ & 4 & $\begin{array}{l}\text { (1) Both intake valve clearance and Exhaust valve } \\
\text { clearance are small; } \\
\text { (2) Intake valve clearance is too small; } \\
\text { (3) Both intake valve clearance and Exhaust valve } \\
\text { clearance are too large; } \\
\text { (4) normal clearance. }\end{array}$ \\
\hline
\end{tabular}

(2) Validation method

10 runs of 10 -folds $\mathrm{CV}$ test for comparing the classifier performances. Each datasets are divided into 10 almost equal-sized blocks randomly, and in each validation, one block was used for test data and the remaining blocks were used for training classifiers. Average all 10 runs as the final results like table $\mathrm{V}$.

(3) Measurement

Throughout all the tests, we measured the classification error rate, i.e., the percentage of incorrectly classified instances.

TableIV. partial result of mixed conditions discretization

\begin{tabular}{|c|c|c|c|c|}
\hline $\mathrm{nn}$ & $\max 1$ & $\operatorname{amax} 1$ & adpmax & class \\
\hline (1796.37-1799.17] & (76.30-76.98] & (5.95-6.45] & $(6.05-6.25]$ & $\mathrm{B}$ \\
\hline (1796.37-1799.17] & $(75.37-76.30]$ & $(6.45-6.75]$ & (7.45-inf) & $\mathrm{B}$ \\
\hline (1796.37-1799.17] & $(76.30-76.98]$ & $(4.65-5.25]$ & $(6.05-6.25]$ & B \\
\hline (1796.37-1799.17] & (76.98-77.45] & $(4.65-5.25]$ & $(6.25-6.55]$ & $\mathrm{B}$ \\
\hline (1782.78-1791.25] & (78.29-inf) & (5.95-6.45] & $(5.55-5.75]$ & A \\
\hline (1782.78-1791.25] & (77.45-78.29] & $(6.75-7.15]$ & $(5.75-6.05]$ & A \\
\hline (1782.78-1791.25] & (77.45-78.29] & $(4.65-5.25]$ & $(5.55-5.75]$ & $\mathrm{A}$ \\
\hline (1782.78-1791.25] & (76.98-77.45] & $(4.65-5.25]$ & $(3.55-5.05]$ & A \\
\hline (1782.78-1791.25] & (77.45-78.29] & $(5.25-5.95]$ & $(5.05-5.55]$ & A \\
\hline (1791.25-1796.37] & (77.45-78.29] & $(6.45-6.75]$ & $(5.75-6.05]$ & A \\
\hline (1796.37-1799.17] & $(75.37-76.30]$ & $(5.25-5.95]$ & $(6.55-7.45]$ & $\mathrm{C}$ \\
\hline (1796.37-1799.17] & $(75.37-76.30]$ & $(6.75-7.15]$ & (7.45-inf) & $\mathrm{C}$ \\
\hline (1796.37-1799.17] & $(-\inf -75.37]$ & (7.15-inf) & $(6.55-7.45]$ & $\mathrm{C}$ \\
\hline (1796.37-1799.17] & $(75.37-76.30]$ & (7.15-inf) & (7.45-inf) & $\mathrm{C}$ \\
\hline (1796.37-1799.17] & $(76.30-76.98]$ & $(6.75-7.15]$ & (7.45-inf) & $\mathrm{C}$ \\
\hline (1806.1-1808.80] & $(76.30-76.98]$ & (7.15-inf) & $(3.55-5.05]$ & $\mathrm{D}$ \\
\hline (1806.1-1808.80] & (76.98-77.45] & (7.15-inf) & $(6.25-6.55]$ & $\mathrm{D}$ \\
\hline (1806.1-1808.80] & (-inf-75.37] & (7.15-inf) & (7.45-inf) & $\mathrm{D}$ \\
\hline (1806.1-1808.80] & (76.30-76.98] & $(6.75-7.15]$ & $(6.25-6.55]$ & $\mathrm{D}$ \\
\hline
\end{tabular}

I.J. Image, Graphics and Signal Processing, 2011, 1, 10-16 


\begin{tabular}{lllll}
\hline$(1806.1-1808.80]$ & $(76.30-76.98]$ & $(7.15-i n f)$ & $(7.45-i n f)$ & $\mathrm{D}$ \\
$(1806.1-1808.80]$ & $(76.30-76.98]$ & $(5.25-5.95]$ & $(6.55-7.45]$ & $\mathrm{D}$ \\
\hline
\end{tabular}

Error rate $=$ number of incorrectly classified instances / the total number of instances of prediction. (4) Comparison algorithms

This paper compares the proposed method with AODE, WAODE and LODE in tableVI.

\section{B. Experimental results and Analysis}

The experimental results are shown in table 4 . Since the average error rates of different classifiers are very close. So we compare each two algorithms A-B via two tailed t-test with a 95 percent confidence level. The results are shown in table 5, where win indicates that algorithm A obtained significantly lower average error rate than algorithm $\mathrm{B}$, draw indicates that $\mathrm{A}$ and $\mathrm{B}$ haven't significant differences, and loss indicates that A obtained significantly higher average error rate than algorithm B. From table VII, we can see that WSPODE outperforms AODE on 9 datasets, outperforms WAODE on 8 datasets, and outperforms LODE on 8 datasets.

TableV. 10 runs error rate of diesel engine data2

\begin{tabular}{cccccc}
\hline dataset & runs & AODE & WAODE & LODE & WSPODE \\
\hline data2d & 1 & $8.06 \%$ & $8.12 \%$ & $7.81 \%$ & $8.12 \%$ \\
data2d & 2 & $7.62 \%$ & $7.44 \%$ & $7.31 \%$ & $7.81 \%$ \\
data2d & 3 & $7.69 \%$ & $7.81 \%$ & $7.56 \%$ & $7.94 \%$ \\
data2d & 4 & $8.00 \%$ & $7.75 \%$ & $7.69 \%$ & $7.81 \%$ \\
data2d & 5 & $7.56 \%$ & $7.56 \%$ & $7.63 \%$ & $7.75 \%$ \\
data2d & 6 & $7.81 \%$ & $7.94 \%$ & $7.50 \%$ & $7.81 \%$ \\
data2d & 7 & $8.13 \%$ & $8.00 \%$ & $7.57 \%$ & $8.00 \%$ \\
data2d & 8 & $7.75 \%$ & $7.75 \%$ & $7.69 \%$ & $7.82 \%$ \\
data2d & 9 & $7.69 \%$ & $7.31 \%$ & $7.37 \%$ & $7.75 \%$ \\
data2d & 10 & $7.56 \%$ & $7.63 \%$ & $7.75 \%$ & $7.44 \%$ \\
& mean & $7.79 \%$ & $7.73 \%$ & $7.59 \%$ & $7.83 \%$ \\
\hline
\end{tabular}

TableVI. detailed results of error rate and standard deviation

\begin{tabular}{ccccc}
\hline dataset & AODE & WAODE & LODE & WSPODE \\
\hline breast_w & $3.02 \pm 0.17$ & $2.98 \pm 0.11$ & $2.95 \pm 0.17$ & $2.92 \pm 0.18$ \\
car & $8.09 \pm 0.36$ & $8.92 \pm 0.29$ & $8.04 \pm 0.35$ & $7.73 \pm 0.43$ \\
diabetes & $21.69 \pm 0.28$ & $21.81 \pm 0.36$ & $21.60 \pm 0.34$ & $21.62 \pm 0.37$ \\
Flags & $39.82 \pm 1.20$ & $39.77 \pm 1.36$ & $41.04 \pm 0.99$ & $39.31 \pm 1.24$ \\
flare & $19.80 \pm 0.57$ & $18.20 \pm 0.25$ & $19.41 \pm 0.68$ & $18.08 \pm 0.31$ \\
heart_h & $14.59 \pm 0.38$ & $14.52 \pm 0.55$ & $14.55 \pm 0.36$ & $14.62 \pm 0.37$ \\
iris & $6.54 \pm 0.84$ & $6.88 \pm 0.72$ & $6.54 \pm 0.84$ & $6.08 \pm 0.76$ \\
lymph & $14.77 \pm 0.85$ & $13.41 \pm 1.38$ & $14.83 \pm 0.97$ & $13.54 \pm 1.46$ \\
machine & $9.48 \pm 0.49$ & $9.24 \pm 0.50$ & $9.48 \pm 0.58$ & $9.43 \pm 0.60$ \\
promoter & $10.93 \pm 1.47$ & $10.03 \pm 2.13$ & $23.22 \pm 2.27$ & $9.77 \pm 1.81$ \\
sonar & $14.22 \pm 0.90$ & $13.21 \pm 0.78$ & $14.32 \pm 0.78$ & $13.84 \pm 0.99$ \\
tic_tac_toe & $25.88 \pm 0.39$ & $27.05 \pm 0.49$ & $25.73 \pm 0.35$ & $25.62 \pm 0.36$ \\
vote & $5.58 \pm 0.16$ & $5.70 \pm 0.18$ & $5.60 \pm 0.16$ & $5.65 \pm 0.19$
\end{tabular}

vowel $\quad 13.04 \pm 0.5715 .94 \pm 0.5210 .76 \pm 0.6112 .60 \pm 0.49$ waveform $13.73 \pm 0.1313 .64 \pm 0.1415 .20 \pm 0.4013 .69 \pm 0.15$ $\begin{array}{lllll}\text { zoo } & 5.21 \pm 0.91 & 5.21 \pm 0.91 & 5.21 \pm 0.91 & 5.13 \pm 0.90\end{array}$ Diesel1 $\quad 17.52 \pm 0.2217 .54 \pm 0.2917 .11 \pm 0.3317 .33 \pm 0.30$ Diesel2 $14.26 \pm 0.2913 .57 \pm 0.2614 .09 \pm 0.2613 .84 \pm 0.31$ Diesel3 $18.60 \pm 0.2919 .01 \pm 0.2018 .32 \pm 0.3218 .38 \pm 0.17$ Diesel4 $17.05 \pm 0.2617 .03 \pm 0.28 \quad 17.22 \pm 0.21 \quad 16.95 \pm 0.26$ $\begin{array}{lllll}\text { Diesel5 } & 8.06 \pm 0.15 & 8.16 \pm 0.19 & 8.13 \pm 0.14 & 7.98 \pm 0.12\end{array}$ mean $\quad 14.38 \pm 0.5214 .37 \pm 0.5714 .92 \pm 0.5714 .00 \pm 0.56$

TableVII. the compared results of two-tailed t-test

\begin{tabular}{ccccccc}
\hline \multirow{6}{*}{ id } & WSPODE WSPODE WSPODE & LODE & LODE & WAODE \\
& -AODE & -WAODE & -LODE & -AODE & WAODE & -AODE \\
\hline 1 & win & draw & draw & win & draw & draw \\
2 & win & win & win & draw & win & loss \\
3 & draw & draw & draw & draw & draw & draw \\
4 & draw & draw & win & loss & loss & draw \\
5 & win & draw & win & draw & loss & win \\
6 & draw & draw & draw & draw & draw & draw \\
7 & draw & win & draw & draw & win & loss \\
8 & win & draw & win & draw & loss & win \\
9 & draw & draw & draw & draw & draw & draw \\
10 & draw & draw & win & loss & loss & draw \\
11 & draw & win & draw & draw & loss & win \\
12 & win & win & draw & win & win & loss \\
13 & draw & draw & draw & draw & draw & draw \\
14 & win & win & win & win & win & loss \\
15 & draw & draw & win & loss & loss & draw \\
16 & draw & draw & draw & draw & draw & draw \\
17 & win & win & loss & win & win & draw \\
18 & win & loss & win & win & loss & win \\
19 & win & win & draw & win & win & loss \\
20 & win & draw & win & loss & loss & draw \\
21 & draw & win & win & draw & draw & draw \\
total & $10 \backslash 11 \backslash 0$ & $8 \backslash 12 \backslash 1$ & $10 \backslash 10 \backslash 1$ & $6 \backslash 11 \backslash 4$ & $6 \backslash 7 \backslash 8$ & $4 \backslash 12 \backslash 5$ \\
\hline & & & & & &
\end{tabular}

\section{CONCLUSIONS}

In order to improve artificial intelligence diesel engine diagnosis accuracy. This paper relaxed the assumption so as to gain smaller classification error rate, took the study of one-dependence estimator, and proposed a new strategy. It took advantage of the diversity and its corresponding NB to weight the SPODEs. Through the comparison experiments with the existing AODE, WAODE and LODE by University of California Irvine (UCI) and 5 diesel engine datasets collected by our lab, the results verified the effectiveness of the proposed algorithm.

\section{REFERENCE}

[1] Sahami M. Learning limited dependence Bayesian classifiers. Proceedings of the 2nd international conference on knowledge discovery and data mining, $\mathrm{pp}$ 334-338, 1996.

[2] E. Keogh, M. Pazzani. Learning Augmented Bayesian Classifiers: A Comparison of Distribution-Based and Classification-Based Approaches. Proc. Int'l Workshop Artificial Intelligence and Statistics, pp. 225-230, 1999. 
[3] N. Friedman, D. Geiger, M. Goldszmidt. Bayesian Network Classifiers. Machine Learning, vol. 29, pp. 131-163, 1997.

[4] Jiang, L., Zhang, H., Cai, Z., Su, J. One Dependence Augmented Naive Bayes. In: Li, X., Wang, S., Dong, Z.Y. (eds.) ADMA 2005. LNCS (LNAI), vol. 3584, pp.186-194. Springer, Heidelberg, 2005.

[5] G.I. Webb, J. Boughton, Z. Wang. Not So Naive Bayes: Aggregating One-Dependence Estimators. Machine Learning, vol. 58, pp. 5-24, 2005.

[6] Jiang L., Zhang H. Weightily averaged one-dependence estimators. In: Yang Q.,Webb G.(eds.) PRICAI2006.LNCS(LNAI), vol.4099, pp970-974, Springer, Heidelberg, 2006.

[7] Liangxiao Jiang, Harry Zhang, and Zhihua Cai. A Novel Bayes Model: Hidden Naïve Bayes. IEEE Transactions on knowledge and data engineering, VOL. 21, NO.10, October 2009.

[8] Flores MJ, Gamez JA, Martinez AM, Puerta JM. HODE: Hidden One-Dependence Estimator. 10th European Conference on Symbolic and Quantitative Approaches to Reasoning with Uncertainty, 5590, 481-492, Verona, ITALY, JUL 01-03, 2009.

[9] Yang, Y., Korb, K., Ting, K.-M., Webb, G.I.: Ensemble Selection for SuperParent-One-Dependence Estimators. In: Zhang, S., Jarvis, R. (eds.) AI 2005. LNCS, vol. 3809, pp. 102-112. Springer, Heidelberg, 2005.

[10] Li N, Jiang Y, Zhou Z. H. Model-Likelihood Based SuperParent-One-Dependence Estimator Ensemble Method. PR\&AI. Vol20, No6, dec 2007.

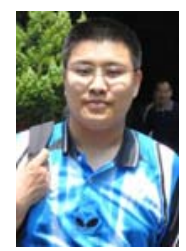

Wang Xin,(1981-), Male, PHD Student, Engaged in the research of intelligent fault diagnosis technique.

October 10, 1981 Xin was born in Dalian City, Liaoning Province. Who graduated from Dalian University in 2004, Computer Science and Technology specialized field and with a Bachelor degree. From 2005 to 2007, Xin studied in Dalian University of Technology and received a master's degree of Computer, Who became a marine engineering PHD student. in Dalian Maritime University at September 2008 and Engaged in the research of intelligent fault diagnosis technique. Xin's papers includes: (1)Wang Xin,Yu Hongliang, Zhang Lin et al. Improved Genetic Algorithm Neural Network Method and the Application in Valve Fault Diagnosis of Diesel Engine.[C] IEEE The 2010 International Conference on Information Security and Artificial Intelligence. (2)Zhang Lin, Wang Xin, Yu Hongliang, Research and Application on Improved Naive Bayesian Classifier Method, IEEE ICIECS2010。 (3)Wang Xin, Yu Hongliang, Zhang Lin et al, Improved Naive Bayesian
Classifier Method and the Application in Diesel Engine Valve Fault Diagnostic IEEE ICMTMA。

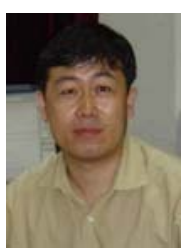

Yu Hongliang,(1963-), Male, professor, $\mathrm{PhD}$ director, Engaged in the research of Power machine fault diagnosis technique.

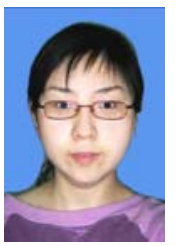

Zhang Lin, (1981-),Female, Lecturer, Master, Engaged in the research of packaging reliability analysis based on artificial intelligence

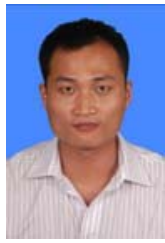

Huang Chaoming,(1982-), Male, PHD Student, Engaged in the research of vibration signal fault diagnosis technique.

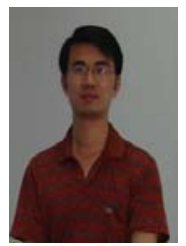

Song Yuchao,(1981-), Male, professor, $\mathrm{PhD}$ director, Engaged in the research of power machine vibration fault diagnosis technique. 